\title{
Formación De Licenciados En Matemáticas En Competencias Para La Enseñanza De La Física.
}

\author{
MG. Alejandro Bolívar Suárez \\ Universidad Pedagógica y Tecnológica de Colombia
}

\begin{abstract}
This article shows the results obtained from the development of a Classroom Project (PA), aimed at the training of Future Math teachers in Mathematics, to be proficient in the performance as teachers in the area of Physics in secondary education. It's analyzed two surveys applied to future graduates and middle school students, who have participated in the semiannual socialization of the advances of the PA, highlighting the benefits of the development of class activities and human resource training with the application of this modality of projects.
\end{abstract}

Keywords: Classroom project, Teaching Physics, Human Resource in Physics

\section{Introducción}

Los proyectos de Aula (PA), se han convertido en una eficiente herramienta didáctica para desarrollar procesos de enseñanza-aprendizaje. No tiene limitaciones en los grados de escolaridad ni en el área del conocimiento. En abundancia se ha cuestionado, la eficiencia de la educación tradicional, fundamentada en la clase magistral con la actitud pasiva de los alumnos y los procedimientos meramente repetitivos de conocimientos elaborados por supuestos eruditos en cada tema. El fracaso del sistema tradicional, se puede resumir en dos actores principales: El primero, son los docentes, porque muestran en muchos casos, total indiferencia al cambio que implica, asumir el reto de nuevas estrategias para mejorar su actividad docente. En segundo lugar, las políticas educativas de los gobiernos, carentes de una visión futurista, basada en la formación integral de su juventud.

Cada campo del conocimiento científico que se imparte en la formación de nuestra juventud en los diferentes niveles de escolaridad, deben ser impartidos por profesionales competentes en cada rama del saber, con alto compromiso ético, que implica una cualificación permanente apoyada por los diferentes gobiernos. Con frecuencia nos quejamos de la mala preparación de los alumnos que recibimos en un curso, de su desmotivación por el estudio o la falta de métodos para asimilar el conocimiento. No puede ser ésta situación una disculpa para no hacer nada. (Michel Picquart, 2007; 2). La enseñanza de las ciencias y en particular de la Física, debe ser una actividad de investigación, fundamentada en la solución de problemas relacionados con la explicación de fenómenos físicos del mundo cotidiano. De ésta forma, estimularemos el desarrollo profesoral y superaremos la formación de nuestros alumnos, basada en la simple transmisión de conocimientos a través de fórmulas mágicas. (Antonio García-Carmona, 2009; 3).

\section{Descripción del problema}

La Física es una ciencia fundamental para el desarrollo científico y tecnológico de un país. En Colombia, aparece esta asignatura como una componente del bagaje de conocimientos que deben poseer nuestros estudiantes que culminan los estudios de la educación media. Quienes hemos tenido la oportunidad de compartir con alumnos en los primeros semestres de la educación universitaria, podemos afirmar que la gran mayoría no ha podido asimilar los fundamentos de Física recorridos en el bachillerato. Los alumnos manifiestan, que no han logrado entenderpara qué sirve la Física, les parece un recetario de fórmulas matemáticas, los docentes que han orientado la asignatura son de áreas diferentes a Física y en muchos casos fue desarrollada solamente conenfoque teórico o simplemente la ven como una asignatura difícil. En contexto, no se tiene el profesorado necesario y suficientemente preparado para orientar la asignatura de Física en la educación media, lo cual se refleja en las argumentaciones descritas anteriormente por los alumnos bachilleres. Complementario con lo anterior, algunos docentes justifican el desarrollo solamente teórico de la asignatura, debido a la baja intensidad horaria de la misma, o a la carencia de laboratorios de experimentación. 
Se plantea entonces la siguiente pregunta de investigación problemática: ¿Cómo contribuir al mejoramiento de la enseñanza de la Física, partiendo de la formación del recurso humano representado en los futuros Licenciados de Matemáticas de la Universidad Pedagógica y tecnológica de Colombia (UPTC)?.

\section{Objetivo general}

Complementar la formación de Licenciados en Matemáticas de la UPTC, en competencias relacionadas con la enseñanza de la Física, para que tengan un eficiente desempeño tanto en la enseñanza de la Física como de la Matemática.

\section{Objetivos específicos}

- Contribuir con el mejoramiento de la enseñanza de la Física, a nivel local, regional y nacional, aportando recurso humano de excelente calidad.

- Desarrollar competencias en Física teórica y experimenta básica en los Licenciados en Matemáticas, aplicables en los diferentes centros educativos de la educación media.

- Orientar al recurso humano en formación, en la habilitación de laboratorios de experimentación real y virtual, para que el recurso económico no sea una limitante en el momento de ejercer la docencia.

- Socializar el trabajo realizado por cada grupo de trabajo en cada semestre, en algún centro educativo de educación media, preferiblemente ubicados en regiones apartadas en la UPTC.

\section{Justificación}

Para mejorar los procesos en la enseñanza de la Física, se requiere de un esfuerzo de varios actores: De las políticas de estado, para formar profesionales idóneos en todos los campos del conocimiento que permita cubrir las necesidades que demanda impartir una educación de calidad y pertinencia. En nuestro país, se da un desequilibrio en el número de docentes en las diferentes áreas del conocimiento, abundando en unas y escaseando en otras. Es así como muchos docentes, tienen que desempeñarse en áreas que no son de su competencia, con el fin de obtener una oportunidad de trabajo, reflejándose el efecto en la formación deficiente de bachilleres. Los docentes juegan un papel primordial para mejorar los procesos en la enseñanza de la Física, deben fundamentar su enseñanza en actividades de investigación, que a su vez, se traduzca en desarrollo profesional de los mismos. Antonio García-Carmona (2009, 3). Desde hace más de una década, se viene imponiendo la figura del profesor investigador, la cual cobra vigencia en la actualidad, para mejorar el nivel de calidad de nuestra educación. Elliott, J((Morata, $4^{a}$ ed., Madrid, 2000). Se debe romper con el esquema de la clase tradicional, basada en clases magistrales con poca conexión entre la teoría y la práctica, sin relación con la vida cotidiana. Motivar a través de hechos reales como las actividades de docencia a través de PA, permitirá vencer el desinterés por el aprendizaje de la Física, que no solo da en Colombia sino en todo el mundo. Jordi Solbes (2007, 92). En una propuesta de trabajo, de formación continua de Licenciados en Matemáticas, se les complementará su formación académica a través del desarrollo de proyectos de aula basadas en la problemática de la enseñanza de la Física en la educación media. De esta forma, se le proporcionará a la comunidad educativadel la región y el país, un recurso humano, preparado para enseñar la Física con base en la investigación de problemas, aplicación de la Física en la ciencia y la tecnología, diseño y elaboración de material de laboratorio para una mejor comprensión de los fenómenos físicos, uso de simuladores como una herramienta didáctica de experimentación virtual y organización y realización de eventos de carácter científico que estimule el interés por las ciencias naturales.

\section{Población Beneficiada}

Los beneficiados por este proyecto, es en general la comunidad educativa del país, representada por los estudiantes de la educación media, las instituciones educativas que podrán contar con docentes idóneos tanto en el campo de la Matemática como de la Física, los mismos licenciados en Matemáticas, que verán en el desarrollo del proyecto una oportunidad adicional en el campo laboral, la UPTC. Porque contribuye en forma efectiva al mejoramiento de la calidad de la educación a través de sus egresados y finalmente el gobierno nacional al disponer de docentes idóneos en el campo de la Física, que minimice la improvisación en la asignación de docentes de otras áreas para la cátedra de Física.

\section{Marco Teórico}

Los temas de Física que se revisan y organizan para desarrollar actividades de clase por parte de los grupos de trabajo del PA, corresponden a los contenidos temáticos que se imparten en los cursos 10 y 11 de la educación media Colombiana. Se resumen estos contenidos en: 
Introducción a la Física, Introducción a vectores, Cinemática de una partícula, Dinámica de una partícula, Mecánica de fluidos, Fundamentos básicos de la Termodinámica, Oscilaciones mecánicas, Ondas mecánicas, Mecánica de fluidos, Óptica geométrica, La carga eléctrica, Circuitos con capacitores y resistores eléctricos, Fundamentos de magnetismo, Ondas electromagnéticas, Aplicaciones de la Física en diferentes campos de la ciencia y la tecnología. Las principales fuentes bibliográficas recomendadas para la revisión de los temas anteriores son: Robert, R., David, H., Kenneth-S, K. (2001); Marcelo, A y Edward-J, Finn. (1995). Se complementa las consultas bibliográficas con las siguientes direcciones electrónicas, que corresponden a los simuladores utilizados en Física, para realizar actividades de apoyo docente en la modalidad de experimentación virtual. Applests Java de Física (Java 1.4)-Walter Fend, Simulaciones Phet. Para el correcto funcionamiento de los simuladores, se debe instalar en los computadores el software Java. Java es un lenguaje de programación y una plataforma informática comercializada por primera vez en 1995 por Sun Microsystems. Se puede tomar en forma gratuita de Internet.

\section{Metodología de Trabajo.}

En cada semestre de labores académicas en la UPTC, se ofrecen dos cursos para cubrir la jornada diurna y nocturna con número variable de estudiantes de la Licenciatura en Matemáticas, en la asignatura de Electiva de profundización II. Los alumnos, que cursan la asignatura, han aprobado los niveles de Física I y II, correspondientes a fundamentos de Mecánica de una partícula y fundamentos de Electromagnetismo respectivamente. El desarrollo de la asignatura Electiva de profundización II, se realiza en la modalidad de PA. Una primera actividad, es socializar en cada grupo la problemática de la enseñanza de la Física en la educación media, recordando vivencias personales y recientes de cada miembro de cada grupo. Se analiza la importancia de mejorar la enseñanza de la Física en la educación media, adquiriendo las competencias necesarias para hacerlo como futuros docentes de Matemáticas y Física. Se indica la importancia que tiene el hecho de que nuestra juventud reciba una mejor educación y nuestros licenciados un campo laboral más amplio. Los temas a cubrir en el desarrollo del PA cada semestre, son los referenciados en el marco teórico, los cuales se distribuyen en los dos grupos, divididos a su vez en subgrupos. Cada subgrupo se encarga de una temática durante todo el semestre, cumpliendo con cuatro etapas. Se trabaja con una intensidad horaria semanal de 4 horas durante todo el semestre para cada grupo. Un 30\% de la intensidad horaria, se dedica para fundamentar a los futuros licenciados en tópicos de Física experimental, uso de software de apoyo docente y elaboración de material de experimentación, para la implementación de un aula de laboratorio de Física en una institución de educación media. El tiempo restante, se dedica a desarrollar las cuatro etapas que se describen a continuación.

\section{- Etapa uno}

Elaboración de un documento escrito, que contenga los fundamentos teóricos de cada tema, su relación y aplicación con la vida cotidiana y su complemento de aprendizaje apoyado en un simulador de fenómenos físicos. Se socializa ante cada grupo de trabajo.

\section{- Etapa dos}

Elaboración de una presentación en PowerPoint de cada tema, que resume el documento escrito, complementada con experimentos caseros. Se socializa ante cada grupo de trabajo.

\section{- Etapa tres.}

Montaje de un poster, que resume las dos etapas anteriores. Se socializa ante cada grupo de trabajo, acompañado de las herramientas experimentales reales y virtuales empleadas en las etapas anteriores.

\section{- Etapa cuatro.}

Se realiza una actividad de socialización de todos los trabajos realizados por los dos grupos, ante una institución de educación media, para los dos últimos grados del bachillerato. Con preferencia, la institución debe estar alejada de la UPTC, con el fin de favorecer a los estudiantes con situación geográfica desfavorable respecto a los centros educativos de las ciudades grandes.

\section{Recolección de la Información.}

Todos los trabajos realizados por los diferentes subgrupos, se recopilan en una carpeta, que constituye un valioso material de apoyo docente para cada uno de los futuros Licenciados. El material, también quedará a disposición de la institución educativa organizadora de la socialización final del PA semestral y de los estudiantes de la educación media participantes en el evento. 


\section{Resultados obtenidos.}

El PA, se ha desarrollados durante 5 semestres consecutivos ( 2 años y medio) y continuará por un tiempo igual al transcurrido; a esta fecha, se tiene previsto hacer una evaluación de los 5 años transcurridos, teniendo como indicador el desempeño de los licenciados que participaron en el PA y ejercen la docencia en el campo de la enseñanza de la Física. Entre otros resultados, se destacan los siguientes:

1.Evaluación de la asignatura. En los 5 semestres, han cursado la asignatura, Electiva de profundización II en la modalidad PA 105 estudiantes, con un rendimiento aprobatorio del 100\%.

2. Encuesta a estudiantes futuros licenciados. Se dispone de una muestra del $20 \%$ del total de alumnos y se basa la encuesta en 7 preguntas, cuyos resultados se resumen a continuación.

Tabla 1. Resumen de la encuesta y sus resultados, aplicada a una muestra de alumnos participantes en el PA.

\begin{tabular}{|l|l|l|}
\hline No. & Pregunta & Resumen de las respuestas \\
\hline 1. & $\begin{array}{l}\text { Aspectos positivos en la formación de } \\
\text { licenciados en Matemáticas que } \\
\text { ofrece la asignatura }\end{array}$ & $\begin{array}{l}\text { Formación de una persona crítica de su propia actividad docente. Ofrece un nuevo } \\
\text { campo de competencia laboral. Se aprende a construir ambientes favorables para la } \\
\text { enseñanza de la Física }\end{array}$ \\
\hline 2. & $\begin{array}{l}\text { Grado de aprendizaje logrado en su } \\
\text { tema }\end{array}$ & $\begin{array}{l}\text { Se logra un grado de aprendizaje significativo. Les agrada tener que consultar. } \\
\text { Destacan la importancia de las herramientas experimentales en el aula }\end{array}$ \\
\hline 3. & $\begin{array}{l}\text { Grado de aprendizaje logrado en el } \\
\text { tema de sus compañeros }\end{array}$ & $\begin{array}{l}\text { Se logra un alto grado de aprendizaje al poder participar de las tareas realizadas con } \\
\text { las ayudas didácticas empleadas por los otros grupos. Se destaca la solvencia con que } \\
\text { cada subgrupo termina sus exposiciones }\end{array}$ \\
\hline 4. & $\begin{array}{l}\text { iEs apropiada la metodología en el } \\
\text { desarrollo de la asignatura? }\end{array}$ & $\begin{array}{l}\text { Destacan la importancia de las clases de Física desarrollando la teoría y la parte } \\
\text { experimental simultáneamente. Genera compromiso para el docente y motivación } \\
\text { para los alumnos. }\end{array}$ \\
\hline 5. & $\begin{array}{l}\text { Experiencia en la actividad } \\
\text { desarrollada con los alumnos de la } \\
\text { educación media }\end{array}$ & $\begin{array}{l}\text { Se encuentran con un ambiente real de lo que será su actividad como futuros } \\
\text { profesionales. Notaron gran motivación, concentración y participación de los } \\
\text { alumnos }\end{array}$ \\
\hline 6. & $\begin{array}{l}\text { Sugiera recomendaciones para } \\
\text { mejorar el desarrollo de la asignatura } \\
\text { en los próximos semestres }\end{array}$ & $\begin{array}{l}\text { Realizar más actividades de socialización en diferentes colegios. Mantener el estilo } \\
\text { de trabajo aplicado en esta asignatura }\end{array}$ \\
\hline 7. & $\begin{array}{l}\text { Evaluar con excelente, bueno, regular } \\
\text { o malo, el desarrollo de la asignatura, } \\
\text { justificando la respuesta }\end{array}$ & $\begin{array}{l}\text { El 95\% contesta que es excelente y el 5\% que es bueno. Argumentan que es una } \\
\text { asignatura que los prepara para su vida laboral y sus proyecciones académicas } \\
\text { futuras. }\end{array}$ \\
\hline
\end{tabular}

3.Encuesta aplicada a una muestra de alumnos de la educación media de diferentes instituciones educativas, que han participado en las actividades de socialización del PA en algún semestre. En la Tabla 2, se observa el contenido de la encuesta y los resultados.

Tabla No. 2. Resumen de la encuesta y sus resultados, aplicada a una muestra de alumnos de la educación media, participantes en los eventos de socialización semestral del PA.

\begin{tabular}{|l|l|l|}
\hline No. & Pregunta & Resumen de las respuestas \\
\hline 1. & $\begin{array}{l}\text { Entre las asignaturas: Física, Matemáticas, } \\
\text { Sociales, humanidades, Química, Biología, Artes, } \\
\text { Idiomas: Elija tres que considere las más } \\
\text { importantes en su formación de bachiller }\end{array}$ & $\begin{array}{l}\text { El 15\% menciona a Física en primer lugar, en segundo y tercer lugar; una } \\
\text { combinación de las demás asignaturas }\end{array}$ \\
\hline 2. & $\begin{array}{l}\text { Cómo considera que le han enseñado la Física (solo } \\
\text { teoría, no le ve aplicación, le parece difícil) }\end{array}$ & $\begin{array}{l}\text { El 20\% reconoce que el profesor se esfuerza en hacerse entender, el 20\% le } \\
\text { parece difícil y el 60\% señala que le han enseñado únicamente fundamentos } \\
\text { teóricos con muchas fórmulas }\end{array}$ \\
\hline 3. & $\begin{array}{l}\text { Cómo le gustaría que le enseñaran las Física (más } \\
\text { intensidad horaria, con más experimentos, etc.) }\end{array}$ & $\begin{array}{l}\text { El 80\% de los alumnos, les gustaría que le enseñaran la Física con más } \\
\text { ayudas experimentales y el 20\%, con experimentos y más intensidad horaria }\end{array}$ \\
\hline 4. & $\begin{array}{l}\text { Qué relación encuentra entre la Física y los } \\
\text { desarrollos científicos y tecnológicos }\end{array}$ & $\begin{array}{l}\text { Resaltan la importancia de la Física para la interpretación de fenómenos } \\
\text { físicos y los adelantos científicos y tecnológicos }\end{array}$ \\
\hline 5. & $\begin{array}{l}\text { Aportes positivos y negativos de la Física para la la } \\
\text { humanidad }\end{array}$ & $\begin{array}{l}\text { El 80\% destaca los aportes positivos de la Física y mencionan algunos, el } \\
20 \% \text { dice que aunque hace aportes positivos también ha hecho malos } \\
\text { aportes, como la bomba atómica }\end{array}$ \\
\hline 6. & $\begin{array}{l}\text { Mencione científicos de la Física y sus } \\
\text { contribuciones importantes }\end{array}$ & $\begin{array}{l}\text { Destacan a Newton y sus leyes fundamentales, Einstein y su teoría de la } \\
\text { relatividad, Arquímedes y sus principios en fluidos, Kepler entre otros }\end{array}$ \\
\hline 7. & $\begin{array}{l}\text { Considera que la carrera que piensa seguir tiene } \\
\text { algo de relación con la Física, o evita que tenga } \\
\text { relación con ella }\end{array}$ & $\begin{array}{l}\text { El 80\% señala que la Física tiene relación con lo que piensa estudiar y el } \\
20 \% \text { que no la tiene. Algunos piensan que no la pueden evadir }\end{array}$ \\
\hline
\end{tabular}

4. Reconocimientos escritos por las instituciones de educación media, en donde se ha adelantado la socialización final de los trabajos PA semestrales. En ellos las instituciones agradecen a la UPTC haberles brindado a sus alumnos, una oportunidad de realimentación de conocimientos en el campo de la Física y destacan el valor que tiene el material de apoyo docente que se les suministra. 


\section{Análisis de los resultados}

El primer punto de los resultados pone de manifiesto, que con metodologías apropiadas y herramientas didácticas experimentales, la Física deja de ser una asignatura difícil, al punto de reducir a cero, el número de alumnos reprobados. En el segundo punto, en la encuesta aplicada a los alumnos futuros maestros, se destaca la importancia que le dan ala formación de docentes críticos de sus actividades de aula, el alto grado de aprendizaje que se logra con actividades de clase, en donde las ayudas experimentales virtuales y reales apoyan la comprensión de los fundamentos teóricos. La socialización de los trabajos desarrollados en el PA, en un ambiente real (instituciones de educación media), les inspira confianza para lo que será su profesión como docentes.

En la encuesta aplicada a los estudiantes de educación media, resaltan los siguientes aspectos: son pocos a los que gusta la Física (15\%) y prefieren evadirla en las carreras profesionales que proyectan seguir, es una consecuencia de la baja motivación con que se les ha orientado la materia.

La gran mayoría (80\%), son conscientes de los aportes positivos de la Física en los adelantes científicos y tecnológicos, pero solicita que se enseñe los fundamentos teóricos de la Física con apoyo experimental.

Los reconocimientos expresados por las instituciones de educación media, que han facilitado la socialización final del PA semestral, es una muestra del interés que tienen por contribuir al mejoramiento de la enseñanza de la Física y su importancia en la formación de sus bachilleres.

\section{Proyecciones del PA.}

El PA, orientado a la formación de Licenciados en Matemática,s competentes para orientar la asignatura de Física en la educación media, continuará por tiempo indefinido, haciendo una evaluación global de resultados al cabo de 5 años. Esto permitirá hacer los ajustes correspondientes, para obtener cada vez, más y mejores resultados.

Se establece un compromiso ético con los futuros licenciados, en el que la preparación recibida a través de este proyecto, sea irradiada a colegas del campo laboral, que no han tenido la oportunidad de cualificarse en la enseñanza de la Física; quienes por alguna razón están orientando esta asignatura en la educación media.

Se espera que en los próximos 5 años, se encuentren en el medio laboral, alrededor de 200 licenciados en Matemáticas competentes para enseñar también la Física en la educación media.

\section{Conclusiones}

- Se ha formado un recurso humano en número significativo (105), y se continuara con el proceso de formación del recurso; los cuales contribuirán a un mejoramiento en la enseñanza de la Física a nivel local, regional y nacional.

- La calidad de los trabajos desarrollados por los diferentes grupos del PA, en los 5 semestres evaluados, ponen de manifiesto un alto grado de conocimientos teóricos y dominio de recursos experimentales, que los habilita para ser idóneos como docentes del área de Física a nivel de la educación media.

- Los futuros licenciados, manifiestan su satisfacción porque sus trabajos realizados, sean socializados en ambientes reales semejantes a los que encontrarán en un futuro inmediato, en el ejercicio de su profesión.

- Se detecta gran interés en los alumnos de educación media porque se les enseñe la Física con ayudas experimentales, lo cual pone de manifiesto el impacto que tendrá la ejecución y continuación de este proyecto.

- El ejercicio de socialización de los trabajos realizados en el PA cada semestre, permite integrar a la comunidad educativa externa con la UPTC, facilitando que esta cumpla con su papel de extensión y servicio a la comunidad.

\section{Referencias Bibliográficas}

Alonso, M y Finn, E. (1995). Física voll .Mecanica. Fondo educativo Latinoameticano, S.A.

Antonio, García-Carmona (2009). Investigación en didáctica de la Física: tendenciasactuales e incidencia en la formación del profesorado.Lat. Am. Journal. Physics. Education, 3 (2), 369-375.

Elliott, J.(2000).La investigación-acción en educación. Madrid, España: Ediciones Morata,S,L.

Picquart, M. (2007). ¿Qué podemos hacer para lograr un aprendizajesignificativo de la física? .Lat. Am. Journal. Physics. Education, 2 (1), 29-36.

Resnick, R., Halliday, D y Krane, K. (2001). FisicaVol 1 y 2. Mexico, Mexico: Compañia editorial continental.

Solbes, J., Montserrat, R y Furió, C. (2007). El desinterés del alumnado haciael aprendizaje de la ciencia: implicaciones en su enseñanza. Didáctica de las ciencias Experimentales y sociales, (21), 91-117.

https://phet.colorado.edu/es/simulations/category/physics

www.walter-fendt.de/ph14s/

https://www.java.com/es/download/

52 